Collegiate Trainees Representatives: Drs P. Casey, K. Dwivedi

\section{North East Division}

Chairman: Dr J. M. Roberts

Secretary: Dr D. A. Stephens

Representative Fellow on Council: Dr J. M. Roberts

Representative Member on Council: Dr T. E. Nelson

Executive Committee: Drs J. Blackburn, G. Blessed, P. G Cannon, Isobel Card, N. E. Crumpton, G. A. Dransfield, D. L. F. Dunleavy, H. G. Egdell, J. N. Haworth, Professor I. Kolvin, Dr H. de B. Warren

Collegiate Trainees Representatives: Drs A. Y. Beaini, S. Baugh

\section{North West Division}

Chairman: Dr M. D. Enoch

Secretary: Dr I. Cookson

Representative Fellow on Council: Dr M. Cashman

Representative Member on Council: Dr H. Eaton

Executive Committee: Drs M. Davenport, J. Higgins, S. A. Leslie, S. Lomax, W. P. Maragakis, G. N. Mathur, M. Silverman, A. Theodossiadis, S. Wright

Co-opted: Drs M. W. Forth, A. W. Johnson, S. Shafar

Collegiate Trainees Representatives: Drs F. Margison, T Rajamanickam

\section{Scottish Division}

Chairman: Dr G. C. Timbury

Secretary: Dr W. D. Boyd

Representative Fellow on Council: Dr W. D. Boyd

Representative Members on Council: Drs J. Evans, M. R. Malcolm

Executive Committee: Drs B. R. Ballinger, J. K. Binns, R. Davidson, Professor R. E. Kendell, Drs M. O'Gorman, M. Rennie, and the Chairman or Secretary of each of the Specialist Sub-Sections and Groups in the Division

Co-opted: Drs J. A. Clark, R. A. W. Ratcliff
Collegiate Trainees Representatives: Drs J. Gallagher, I. Pullen

Chairman: Dr R. Maggs

Secretary: Dr K. Hamadah

Representative Fellow on Council: Dr J. A. Hatrick

Representative Member on Council: Dr A. C. Smith

Executive Committee: Drs O. Briscoe, J. Cleobury, G. Davis, E. R. Evans, K. Fraser, J. A. Hatrick, M. Heller, J. M. Hirons, J. Kellett, K. O'Keefe, D. Smedberg, P. Woolf

Collegiate Trainees Representatives: Drs L. Sireling, H. Waters

Chairman: Dr H. Crow

Secretary: Dr C. Davies

Representative Fellow on Council: Dr D. F. Early

Representative Member on Council: Dr C. Davies

Executive Committee: Drs I. G. Christie, D. H. Dick, M. J. Gay, A. L. Horton, J. Jancar, G. E. Langley, J. S. Lyon, H. G. Morgan, P. N. Nott, G. D. P. Wallen, A. E. Wilson Collegiate Trainees Representatives: Drs K. Craig, D. Martin

\section{Welsh Division}

Chairman: Dr R. M. Cuthil

Secretary: Dr A. M. P. Kellam

Representative Fellow on Council: Dr T. Gwynne Williams Representative Member on Council: Dr A. M. P. Kellam

Executive Committee: Drs K. G. Mohan Bhakta, M. J. Craft, T. J. G. Davies, M. Harper, J. M. Hughes, P. Jackson, D. C. Jones, E. M. E. Jones, E. Kapp, A. B. Rolfe, R. Viswanathan, R. J. W. Williams, T. Gwynne Williams

Ex-officio: Dr M. W. Annear, Professor K. Rawnsley, Drs H. Rosenberg, T. T. Westhead

Collegiate Trainees Representatives: Drs P. Marshall, R. Scorer

\title{
OFFICERS OF THE SECTIONS AND GROUPS
}

Psychotherapy Section
Chairman: Dr I. S. Kreeger
Secretary: Dr D. C. James
Representative Fellow on Council: Dr I. S. Kreeger
Representative Member on Council: Dr J. R. Pedder
Executive Committee: Drs M. Aveline, J. Cobb, A. Cooklin,
J. Denford, M. Feldman, M. Glasser, R. H. Gosling, J. R.
Pedder, B. J. Muir, J. Steiner, J. D. Templeton, H. Wolff

Co-opted: Drs J. Evans, M. Hartman, C. J. Lucas, H. Rosenberg,

Observer: Dr L. Brierley

Collegiate Trainees Representative: Dr F. Botica

Forensic Psychintry Section

Chairman: Professor R. S. Bluglass

Secretary: Dr P. Bowden 
Representative Fellow on Council: Dr P. T. d'Orban Representative Member on Council: $\mathrm{Dr}$ J. R. Hamilton

Executive Committee: Drs A. Campbell, B. D. Cooper, Professors T. C. N. Gibbens, J. C. Gunn, Drs H. Hunter, J. A. C. MacKeith, A. McQuaid, H. Milne, I. Pierce James, D. G. A. Westbury, P. G. Woolf

Co-opted: Drs M. Faulk, J. Higgins, R. C. Ingrey-Senn, V. Molony, D. O. Topp

Observer: Dr L. J. F. Warnants

Collegiate Trainees Representative: Dr G. Glancy

\section{Mental Deficiency Section}

Chairman: Dr J. Jancar

Secretary: Dr W. R. McKibben

Representative Fellow on Council: Dr Valerie Cowie

Representative Member on Council: Dr W. R. McKibben

Executive Committee: Drs J. T. Bavin, J. A. Corbett, M. Craft, A. S. R. Goonetilleke, J. A. Hatrick, M. Leyshon, D. M. Lynch, M. Quinn, Gwyn E. Roberts, R. M. Veall, P. Sylvester

Co-opted: Drs F. Broderick, W. I. Fraser, D. A. Primrose, E. W. Shepherd, H. Smyth

Observer: Dr R. Wilkins

Collegiate Trainees Representative: Dr G. Holt

Section for the Psychiatry of Old Age
Chairman: Dr R. A. Robinson
Secretary: Dr P. M. Jefferys
Representative Fellow on Council: Dr R. A. Robinson
Representative Member on Council: Dr P. M. Jefferys
Executive Committee: Professor T. A Arie, Drs K.
Bergmann, G. Blessed, C. Godber, P. Goddard, D. J.
Jolley, G. E. Langley, R. Levy, C. McDonald, G.
Mitchell, R. M. Philpott, B. Pitt, F. Post, P. V. Spencer,
E. R. Taws, C. A. Trotter (Collegiate Trainees
Representative)
Co-opted: Dr J. Fisk
Observer: Dr R. Wilkins

\section{News Items}

\section{Money for Eczema Research}

The Research Fund of the National Eczema Society now stands at around $£ 30,000$ and is open for applications for grants in the region of $£ 1,000$ to $£ 3,000$. The Society is interested in research on all types of eczema and into all aspects of the disease. Applications for funds to assist research in the management of the condition will be given special consideration. Further particulars and instructions on how to apply for a grant may be obtained from the General Secretary, National Eczema Society, 5-7 Tavistock Place, London WC1H 9SR. (Submission date for 1980 is 1 September).

\section{Section of Social and Community Psychiatry}

Chairman: Dr J. P. Leff

Secretary: Dr A. C. Brown

Representative Fellow on Council: Dr J. P. Leff

Representative Member on Council: Dr B. Morris

Executive Committee: Drs J. R. Ashton, D. H. Bennett, A. Brook, J. D. W. Fisher, H. Freeman, Professor S. Hirsch, Drs P. M. Jefferys, J. Lomax-Simpson, B. Morris, C. Parkes, Professor E. Paykel, Drs U. P. Seidel, J. Stuart Whiteley, R. Williams

Observer: Dr A. Sippert

Collegiate Trainees Representative: Dr I. Pullen

\section{Child and Adolescent Paychiatry Section}

Chairman: Dr W. Lumsden Walker

Secretary: Dr H. C. Cameron

Representative Fellow on Council: Dr W. Lumsden Walker

Representative Member on Council: Dr H. C. Cameron

Executive Committee: Drs I. S. Berg, J. M. Black, J. Byng-

Hall, C. L. Casimir, J. Erulkar, E. A. Frommer, A. Gath,

M. J. Gay, Professors P. Graham, I. Kolvin, Drs B.

McCarthy, D. G. Melville Thomas, W. Parry-Jones, P.

Pinkerton, L. G. Scarth, C. Wardle, J. Wells

Co-opted: Drs A. D. Black, E. Kapp, A. R. Nicol, J. Powell, A. M. Smith

Observer: Dr L. Brierley

Collegiate Trainees Representative: Dr Oliver Shanks

\section{Dependence/Addiction Group}

Chairman: Dr Brian Hore

Secretary: Dr M. I. Akhter

Executive Committee: Drs J. Madden, J. Morgan, A.P. Thorley

\author{
Biological Paychiatry Group \\ Chairman: Dr D. Richter \\ Secretary: Dr T. Crow
}

\section{The Department of Mental Handicap at St George's}

A new department of Psychiatry of Mental Handicap has been established at St George's Hospital Medical School, Tooting, London. The Professor, Dr Joan Bicknell, has been appointed and will take up her new post on the 1 May, 1980. Initially there will be two Senior Lecturers in the Psychiatry of Mental Handicap in the department, one providing a clinical service for Kingston and Richmond Area Health Authority with beds at Normansfield, Teddington, and the second providing a clinical service for the Merton, Sutton and Wandsworth Area Health Authority (T), with beds at 